\title{
MULTI-BAND ASYMMETRIC PIEZOELECTRIC MEMS MICROPHONE INSPIRED BY THE ORMIA OCHRACEA
}

\author{
Yansheng Zhang, Ralf Bauer, James F.C. Windmill, and Deepak Uttamchandani \\ University of Strathclyde, Glasgow, UK
}

\begin{abstract}
A multi-band piezoelectric directional MEMS microphone is demonstrated based on a bio-mimetic design inspired by the parasitoid fly Ormia ochracea, using the PiezoMUMPs multi-user foundry process. The device achieves a directional sound field response within four frequency bands, all lying below $15 \mathrm{kHz}$. It acts as a pressure gradient microphone with hyper-cardioid polar patterns in all frequency bands, with the measured mechanical sensitivity being in good agreement with acoustic-structural simulations conducted in COMSOL Multiphysics. The maximum experimentally measured acoustic sensitivity of the device is $19.7 \mathrm{mV} / \mathrm{Pa}$, located at a frequency of $7972 \mathrm{~Hz}$ and sound incidence normal to the microphone membrane.
\end{abstract}

\section{INTRODUCTION}

Directional microphones have been investigated since the 1930s, with the first commercially produced ribbon microphone invented by Harry F. Olson et al. having dimensions of about $30 \mathrm{~cm} \times 10 \mathrm{~cm} \times 6.5 \mathrm{~cm}$ [1]. It provided a cardioid directivity pattern and was widely used in the broadcast and recording industries. With the ever increasing popularity of smartphones and other mobile devices since the 1990s and focus on the development of miniaturized hearing aids, manufacturers of directional microphones not only concentrate on their performance, but also on their size and miniaturization potential. Through this, the development of MEMS directional microphones has become a focus area to both improve device miniaturisation and also offer high acoustic sensitivity and low noise. In general, a directional microphone response can be accomplished by using two or more matched omnidirectional microphones arranged in defined lateral patterns or by using a microphone membrane on which both sides are exposed to the acoustic field. The directionality of the microphone response is achieved by measuring the pressure difference between the omnidirectional microphones or the two surfaces of a membrane with sound field exposure on the front and back. Most of the devices developed so far require complex post-processing after signal acquisition as their dimensions are large enough to cause diffraction and phase interference. Thus, to avoid such complexity, the dimension of the device must be controlled below a certain range. For a directional microphone working below $15 \mathrm{kHz}$ this leads to a mechanically active region of the device less than $3 \mathrm{~mm}$ in radius [2]. However, this results in a time difference of sound arrivals (TDOA) of only $3 \mu \mathrm{s}$ if one assumes that the lateral distance between two omnidirectional microphones is $1 \mathrm{~mm}$.

In order to increase the pressure gradient and improve the sensitivity with respect to accurately locating a sound source, a range of directional MEMS microphones mimicking the hearing mechanism of Ormia ochrecea, a parasitoid fly, have been developed. Due to mechanical coupling between its two tympana, the female Ormia has a great capability of locating the mating calls of its hosts with an angular resolution of $2^{\circ}$, even though the interaural distance is only $520 \mu \mathrm{m}$. Miles et al. developed a series of polysilicon based microphones inspired by this insect, integrating optical sensing or capacitive sensing [3, 4]. Touse et al. built an Ormia-inspired microphone using an asymmetric geometry [5] in which the mechanical response is transduced using electrostatic comb drives. Biomimetic microphones presented by $\mathrm{Yu}$ et al. show a directional acoustic response depending on the mIPD (mechanical inter-aural phase difference) between its two clamped circular membranes [6]. This type of directional microphone was also investigated for two dimensional sound localization [7]. Additionally, Kuntzman et al. introduced piezoelectric sensing into this kind of microphone category [8].

However, all of these previous designs operate at no more than two frequency bands. Out of these, the designs with a closed air cavity behind the microphone membrane have a $\sin \theta$ dependent response on the sound incident angle $\theta$ (with $\theta=0^{\circ}$ being the normal incident on the microphone), which results in lower sound energy incident on the microphone compared to devices having a $\cos \theta$ dependence. The motivation for the research work presented here is to increase the number of working frequency bands, in other words, to expand the total efficiency of the frequency response. Moreover, the new design features piezoelectric sensing capabilities with a maximum acoustic sensitivity at $\theta=0^{\circ}$ combined with a $\cos \theta$ dependent response

\section{DESIGN AND CONCEPT}

The novel biomimetic MEMS microphone design consists of two coupled membranes, arranged in an outer frame and an inner plate configuration, which rotate about a combined off center torsion beam fixed to the substrate. Scanning electron microscope images of the fabricated MEMS microphone design are shown in Figure 1. The overall external dimensions of the microphone are $2.4 \mathrm{~mm} \times 1.06 \mathrm{~mm} \times 10 \mu \mathrm{m}$, with a sound active area of the outer frame of $1.1 \times 10^{-3} \mathrm{~mm}^{2}$ and of the inner frame of $5.8 \times 10^{-4} \mathrm{~mm}^{2}$. The active device area is released through a full backside etch step of a $400 \mu \mathrm{m}$ thick substrate. Each rotating section has two main movement mode shapes (i.e. a rocking mode and a bending mode) as is present in the hearing system of Ormia ochrecea. The acoustic response of the microphone is determined by transduction of the displacement amplitudes generated by the sound pressure gradient between the front and the back of the wings of both membrane parts. The displacement is sensed indirectly through the stress induced in a $500 \mathrm{~nm}$ thick piezoelectric aluminum nitride (AIN) film deposited on sections of the device located close to the torsion 


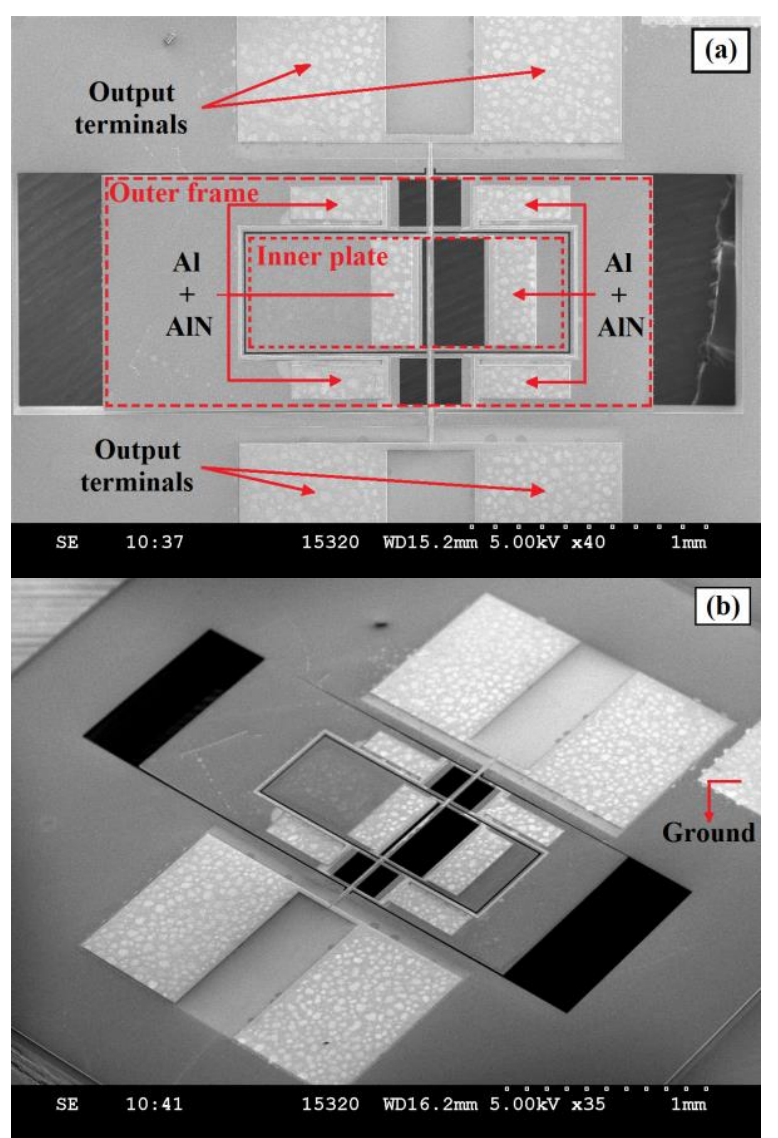

Figure 1: SEM images of the new asymmetric piezoelectric microphone: (a) Top view (b) Perspective view

beam. The devices were fabricated using the PiezoMUMPs multi-user process supplied by MEMSCAP [9]. A $1 \mu \mathrm{m}$ thick aluminum (Al) layer allows electrical routing of the signals created in the piezoelectric sensing locations. An additional thermal oxide layer is available in the fabrication process to electrically isolate the $\mathrm{Al}$ routing from the doped silicon device layer acting as ground port for the piezoelectric sensing layer. Figure 2 shows a cross section of the final layer structure, including the $400 \mu \mathrm{m}$ thick substrate.

The microphone design was chosen based on a lumped element model, which is similar to our previous work [10]. Differing from the previous design, the new device has an asymmetric geometry that results in an unequal mass distribution of the wings in both the outer frame and inner plate. This also results in a variation of the inherent stiffness of each wing and the coupling stiffness between them. The mathematical derivation of the asymmetric lumped element model leads to the following equations, which show the same behavior for all working frequencies. For example, for one of the wings of the outer frame the displacement amplitude $A_{1}$ can be written as:

$A_{1} \propto \cos \theta \sqrt{\left[s_{2} \alpha_{1}-s_{1} K_{t}\right]^{2}+\left(s_{2} d_{2} \alpha_{1}-s_{1} d_{1}\right)^{2} k_{r}^{2} \sin ^{2} \theta}$ $\propto \cos \theta$

with $\alpha_{1}=-\omega_{r}^{2} m_{1}+K_{1}+K_{t}$.

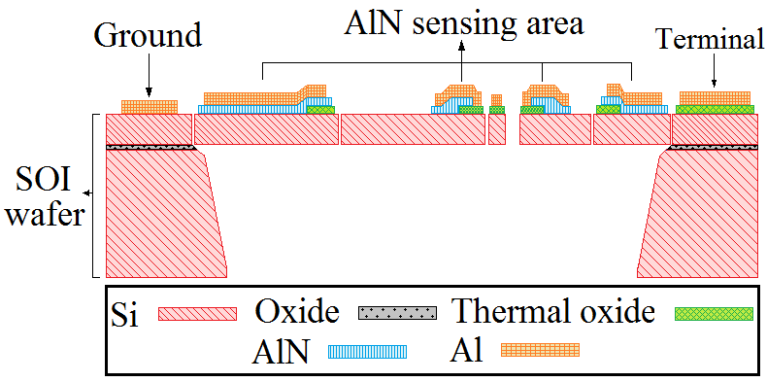

Figure 2: Cross section view of the fabricated device

Here $s_{1}$ and $s_{2}$ are the surface area of the wing under investigation and the second wing respectively, $\mathrm{d}_{1}$ and $\mathrm{d}_{2}$ are the distance between the mass center of each wing and the torsion axis, $K_{1}$ is the stiffness of the wing with mass $\mathrm{m}_{1}, \mathrm{~K}_{\mathrm{t}}$ is the coupling stiffness between both wings, $\mathrm{k}_{\mathrm{r}}$ is the wavenumber of the acoustic excitation at the frequency $\omega_{\mathrm{r}}$. This cosine-dependence behavior holds for both the rocking and bending mode of the two microphone parts, using the respective surface area, excitation frequency, mass and stiffness. In comparison to the previous work which had a symmetric geometry and different directional behavior in all working frequencies, the new device has a uniform directional performance in all four working bands, which reduces the post processing requirements. The $\cos \theta$ dependence also produces exactly the behavior of a first order pressure gradient microphone.

\section{SIMULATION AND CHRACTERIZATION}

The mode shapes of the new design were investigated theoretically using the finite element software COMSOL Multiphysics and experimentally using a Polytec PSV300-F Laser Doppler Vibrometry (LDV) with a close-up scanning head unit. The simulations were created using an acoustic-structure interaction model, evaluating both the mechanical resonance eigenfrequencies of the design and the frequency and angular response to a plane-wave sound incident on the device. In both cases the simulation included the microphone and its $5.5 \mathrm{~mm} \times 5.5 \mathrm{~mm}$ MEMS die.

The experimental setup included a loudspeaker (ESS Heil Air-motion Transformer) driven by a chirp signal with a bandwidth of $15 \mathrm{kHz}$. It was placed 1 metre away from the centre of the microphone to produce a vertical sound wave incident on the front surface of the die. The MEMS chip was bonded on an approximately $4 \mathrm{~cm} \times 2.5 \mathrm{~cm} \times 1.5 \mathrm{~mm}$ PCB in order to build the accessible interface between the sample and the analogue instruments for acquiring the electrical readouts. In addition, a Brüel \& Kjær (B\&K) 4138 pressure-field microphone connected to a preamplifier was placed close to the sample, acting as sound pressure reference.

Figure 3 shows that all four resonance frequencies determined by the LDV are closely matched with the simulations and are located below $15 \mathrm{kHz}$. The outer frame provides the first rocking mode and the first bending mode while the inner plate is responsible for the second rocking mode and second bending mode. The resonances are also shown in Figure 4. Good agreement of the mechanical sensitivity between the simulations and 


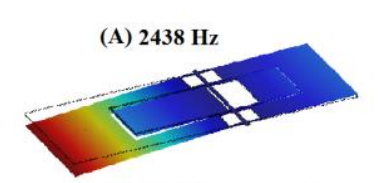

(B) $4909 \mathrm{~Hz}$

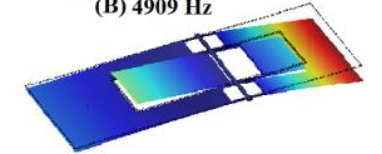

(C) $7923 \mathrm{~Hz}$

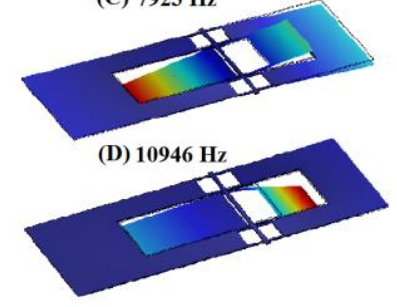

Figure 3: Resonance mode shapes obtained from COMSOL simulations (Left) and Polytec LDV (Right)

measurements reveals that the mechanical performance of the manufactured device conforms to expectation. As is shown in Figure 4, the measured mechanical sensitivity of the larger wing of the outer frame is $3.17 \mu \mathrm{m} / \mathrm{Pa}$ and 1.07 $\mu \mathrm{m} / \mathrm{Pa}$ at $2362 \mathrm{~Hz}$ and $4884 \mathrm{~Hz}$, respectively, while the sensitivity of the larger wing of the inner plate is 1.03 $\mu \mathrm{m} / \mathrm{Pa}$ and $2.76 \mu \mathrm{m} / \mathrm{Pa}$ at $7972 \mathrm{~Hz}$ and $11028 \mathrm{~Hz}$ respectively. The simulation and measurement overlap has only a slight discrepancy at the $2^{\text {nd }}$ resonance due to a none convergent finite element simulation at the exact resonance frequency. As the device has very low damping, most of the measurement noise at low frequencies is background noise.

The electrical output signals are measured using a 60 $\mathrm{dB}$ gain Edmund Optics transimpedance amplifier in combination with a SR850 lock-in amplifier (using a sensitivity of $10 \mathrm{mV}$ and a time constant of $100 \mathrm{~ms}$ ) connected to an oscilloscope. The signals are acquired for both the $\mathrm{X}$ and $\mathrm{Y}$ axis of the lock-in amplifier using an Agilent InfiniiVision oscilloscope. The device under test was mounted on a rotation stage which was positioned at the center of an anechoic box. The B\&K reference microphone is again placed next to the sample to measure the reference sound pressure during the experiment. The device under test is located in the far-field of the loud speaker and its surface is aligned to have a normal sound incidence. The experimental setup for acquiring the electrical signals is Figure 5.

The combination of the electrical signals from the outer frame and inner plate pick up all four frequency bands. The acoustic sensitivity of the first two frequencies is around $5.4 \mathrm{mV} / \mathrm{Pa}$ and $14.8 \mathrm{mV} / \mathrm{Pa}$, with the third and fourth frequency having a sensitivity of around 19.7 $\mathrm{mV} / \mathrm{Pa}$ and $16.8 \mathrm{mV} / \mathrm{Pa}$. Figure 6 shows the acoustic sensitivity spectrum obtained from the terminals corresponding to the larger wings of each rotation section.

Figure 7 presents the measured normalized voltage outputs from the outer frame and the inner angle with varying sound incident angle. The finite element analysis described earlier was also applied for varying sound incident angles. Both the simulation and experimental

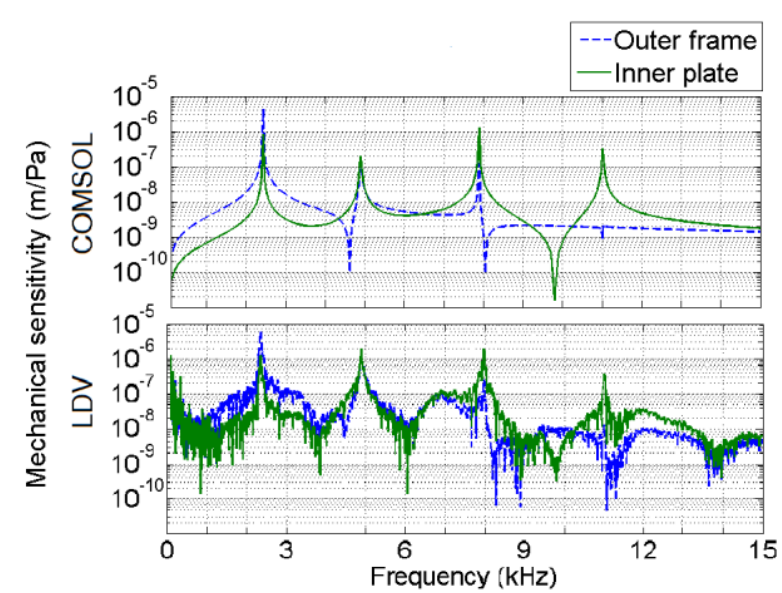

Figure 4: Frequency spectrum of the mechanical sensitivity to sound excitation at $\theta=0^{\circ}$ obtained from the FEM simulations and experimental $L D V$ measurements.

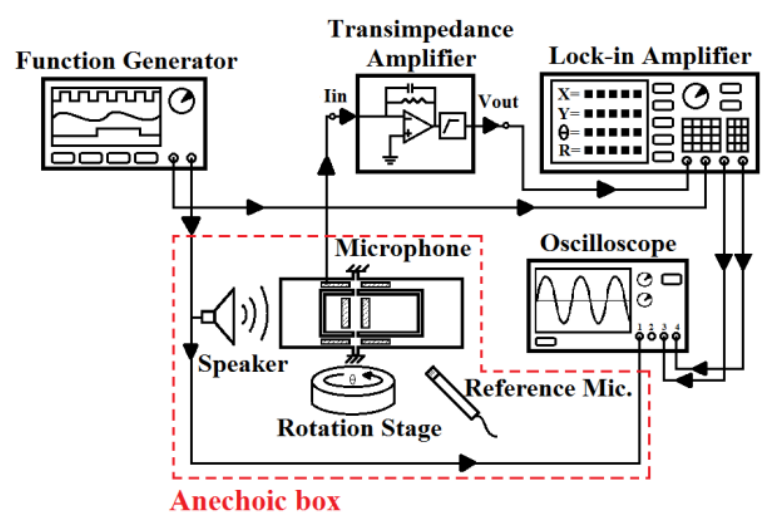

Figure 5: Experimental setup for measuring the piezoelectric voltage response of the microphone to acoustic stimuli with varying incident angles

measurement show that the new device has a bidirectional pattern in all frequency bands. However, while the simulation shows an ideal figure- 8 pattern, the experimental results show a reduced sound response at the back of the device. Its behavior is a hyper-cardioid at all four resonance frequency, especially at the $1^{\text {st }}, 2^{\text {nd }}$ and the $4^{\text {th }}$ resonance. The reduced backside response potentially

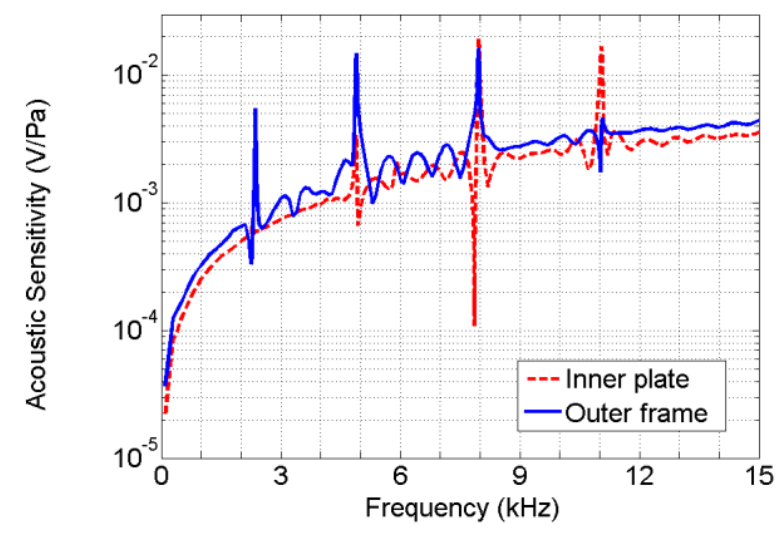

Figure 6: Frequency spectrum of the measured acoustic sensitivity, showing the response of the terminals on the inner plate and the outer frame 

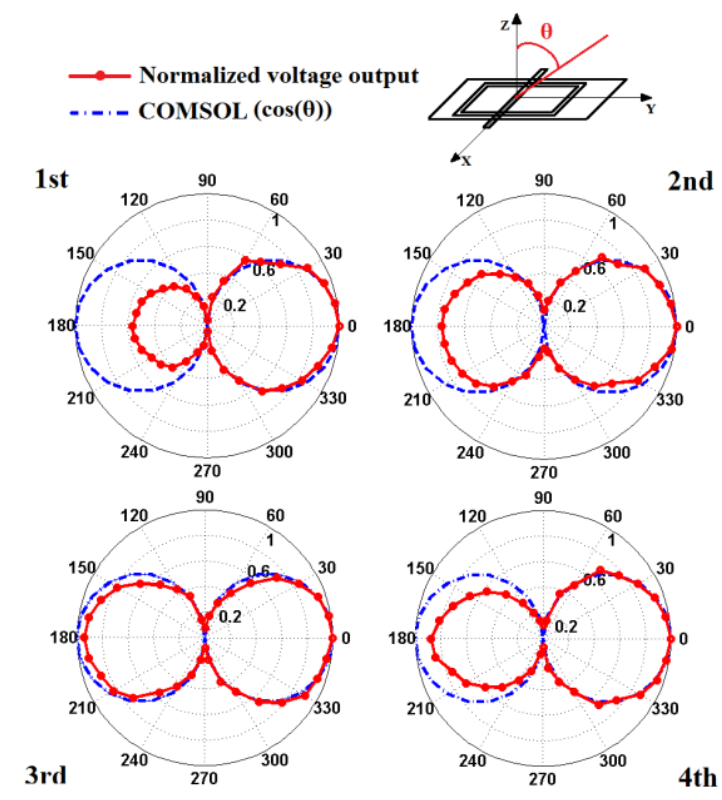

Figure 7: Normalised acoustic directionality patterns at the four resonance frequencies

originates from the sound energy reduction at the backside of the chip due to sound reflection and diffraction occurring in the hole of the PCB. Therefore, the pressure gradient between the two surfaces of the device is smaller when the rear surface faces to the speaker than the front surface does.

\section{CONCLUSION}

The new directional MEMS microphone inspired by Ormia ochracea has a directional sound response in four frequency bands around $2362 \mathrm{~Hz}, 4884 \mathrm{~Hz}, 7972 \mathrm{~Hz}$ and $11028 \mathrm{~Hz}$. It is actuated by the sound pressure gradient between the front and the rear surface of the wings. The electrical readout is achieved using an AlN piezoelectric layer deposited on the four rotation wings of the device. The COMSOL simulation shows that the microphone has an ideal figure- 8 directional pattern at all four resonances, while the experimental results show that the manufactured device performs as a hyper-cardioid microphone at the $1^{\text {st }}$, the $2^{\text {nd }}$, and the $4^{\text {th }}$ resonance. This is mainly because of the sound reflection and diffraction in the hole of the PCB that potentially reduce the sound pressure on the rear surface of the device.

Future work will, investigate the causes for the noise in the electrical outputs and the decrease of amplitude at the $1^{\text {st }}$ resonance. In addition, the sound pressure around the rear surface of the microphone and its influence on the displacement amplitude and directionality of the device will be investigated.

\section{ACKNOWLEDGEMENTS}

The authors would like to thank James Brown at Centre for Microsystems and Photonics in University of Strathclyde for taking SEM images of the finished device. Research described in this paper was in part funded through EPSRC (UK) grant no. EP/M026701/1.

\section{REFERENCES}

[1] J. Weinberger, H. F. Olson and F. Massa, "A Unidirectional Ribbon Microphone”, J. Acoust. Soc. Am., vol. 5, no. 2, pp. 139-147, 1933.

[2] L.E. Kinsler, A. R. Frey, A. B. Coppens, J. V. Sanders, Fundamentals of Acoustics, John Wiley \& Sons, 2000.

[3] R. N. Miles, Q. Su, W. Cui et al., "A low-noise differential microphone inspired by the ears of the parasitoid fly Ormia ochracea", J. Acoust. Soc. Am., vol. 125, pp. 2013-2026, 2009.

[4] R. N. Miles, W. Cui, Q. T. Su, D. Homentcovschi, "A MEMS Low-noise Sound Pressure Gradient Microphone With Capacitive Sensing", J. Microelectromech. Syst., vol. 24, no. 1, 2015.

[5] M. Touse, J. Sinibaldi, K. Simsek et al., "Fabrication of a microelectromechanical directional sound sensor with electronic readout using comb fingers", Applied Physics Letters, vol. 96, no. 17, pp. 1-3, 2010.

[6] H. Liu, L. Currano, D. Gee et al., "Understanding and mimicking the dual optimality of the fly ear", Scientific Reports, vol. 3, 2013.

[7] A. P. Lisiewski, H. J. Liu, M. Yu, "Fly-ear inspired micro-sensor for sound source localization in two dimensions", J. Acoust. Soc. Am., vol. 129, no. 5, pp. 166-171, 2011.

[8] M. L. Kuntzman, N. N. Hewa-kasakarage, A. Rocha et al., "Micromachined In-Plane Pressure Gradient Piezoelectric Microphones", IEEE Sensors J., vol. 15, no. 3, pp. 1347-1357, 2015.

[9] "MEMSCAP | PiezoMUMPs and about its process", http://www.memscap.com/products/mumps/piezomu mps.

[10] Y. Zhang, J. F. C. Windmill, D. Uttamchandani, "Biomimetic MEMS Directional Microphone Structures for Multi-Band Operation", in Proc. IEEE Sensors, Valencia, November 2-4, 2014, pp. 440-443.

\section{CONTACT}

*Y. Zhang, tel: +44-141-444-7411; yansheng.zhang.101@strath.ac.uk 